Article

\title{
Comprehensive Analysis of Antioxidant Compounds from Lippia citriodora and Hibiscus sabdariffa Green Extracts Attained by Response Surface Methodology
}

\author{
María del Carmen Villegas-Aguilar ${ }^{1,2}$, Francisco Javier Leyva-Jiménez ${ }^{2}$, \\ María de la Luz Cádiz-Gurrea ${ }^{1,2, * D}$, Antonio Segura-Carretero ${ }^{1,2,+}$ and \\ David Arráez-Román 1,2,*,+(i) \\ 1 Department of Analytical Chemistry, University of Granada, 18071 Granada, Spain; \\ marivillegas@ugr.es (M.d.C.V.-A.); ansegura@ugr.es (A.S.-C.) \\ 2 Research and Development of Functional Food Centre (CIDAF), 18016 Granada, Spain; jleyva@cidaf.es \\ * Correspondence: mluzcadiz@ugr.es (M.d.l.L.C.-G.); darraez@ugr.es (D.A.-R.) \\ + These authors shared author co-seniorship.
}

Received: 22 October 2020; Accepted: 23 November 2020; Published: 25 November 2020

\begin{abstract}
Phenolic compounds have shown to have a high bioactive potential against various pathologies, postulating as an interesting alternative to manage some diseases. In this sense, both Lippia citriodora and Hibiscus sabdariffa are two botanical sources with a demonstrated high bioactive potential, in which their antioxidant capacity stands out. In this work, the optimization of the extraction conditions for the recovery of phytochemicals from L. citriodora leaves and H. sabdariffa calyces has been carried out using Response Surface Methodologies (RSM) considering their total polar compounds measured by HPLC-ESI-TOF/MS and Folin-Ciocalteu assay, and its antioxidant capacity evaluated by Ferric Reducing Antioxidant Power (FRAP) and Trolox Equivalent Antioxidant Capacity (TEAC) assays. The results showed that to maximize the antioxidant capacity in $H$. sabdariffa, a moderate temperature and high ethanol percentage are needed, while a low temperature and a high percentage of ethanol are needed in L. citriodora. In addition, with the results obtained in the multiple response analysis, it is possible to affirm the importance of this type of analysis to develop functional ingredients, taking into account both total content of phenolic compounds and their bioactivity. Furthermore, as confirmed in this study, these analyses can be extrapolated in different techniques and in different matrices, with phenolic compounds from different families being important to develop new high added value products for food, pharmaceutical or cosmetic industries.
\end{abstract}

Keywords: phenolic compounds; response surface methodology; Lippia citriodora; Hibiscus sabdariffa; antioxidant; Microwave-Assisted Extraction (MAE); Pressurized Fluid Extraction (PLE)

\section{Introduction}

In recent years, both nutraceuticals and functional food have received increasing attention from the scientific community, consumers and food manufacturers [1]. This growing interest is related to their composition and health benefits. These benefits are attributed by the source used during their manufacturing, which are, generally, botanicals. In fact, there is a huge variety of botanicals that have been reported to exhibit biological properties such as antioxidant or anti-inflammatory [2-4]. In this sense, plants such as Hibiscus sabdariffa and Lippia citriodora stand out among an extensive list, since both have been traditionally used to prepare herbal drinks with numerous health benefits. Several of these beneficial properties have been related to the bioactive compounds present in their composition, mainly, phenolic compounds $[5,6]$. 
Several reports of $H$. sabdariffa have demonstrated its potential effects such as antioxidant [6], anti-obesity [7], hypotensive [8], antidiabetic [7], hypocholesterolemic [9], immunomodulatory [10], hepatoprotective [11] and diuretic [10]. In the case of L. citriodora, its antioxidant [10], anti-inflammatory [12], antimicrobial [13] and anticancer [10] properties, among others, have been also highlighted. These biological properties are especially related to the phenolic compounds, which are secondary metabolites contained in their leaves, stems or flowers.

In spite of having great benefits on the human health, the amount of these compounds are usually reduced, being necessary an adequate extraction technique to solve several issues: (i) to avoid or decrease their degradation during the extraction processes and (ii) to concentrate them or to perform selective extractions. There are numerous extraction techniques to attain enriched extracts in phenolic compounds from botanical sources, but currently the non-conventional or advanced techniques stand out, due to the fact that they have fewer extraction times, lesser consumption of solvents (which may be Green and Generally Recognized As Safe, GRAS) and energy. For these reasons, they have been recognized as environmentally friendly techniques, being more efficient than conventional ones [14,15]. Some examples of advanced extraction techniques are Microwave-Assisted Extraction (MAE) and Pressurized Fluid Extraction (PLE).

Regardless of the extraction technique selected, during an extraction process, the adjustment of the extraction parameters is an important step since, depending on the targets desired, the best extraction conditions may have a determinant influence. In the last decade, the use of Response Surface Methodologies (RSM) has been applied with the purpose of optimizing the extraction conditions considering the aims desired, enhancing the efficiency of advanced extraction technologies $[14,16,17]$.

There is a large number of studies that propose different extraction methods to obtain enriched extracts in specific bioactive compounds by RSM and its possible incorporation in functional food or in nutraceuticals [14,17-19]. However, the health benefits of the extracts lie in their bioactivity, which is influenced by synergies and antagonism between compounds. Due to this, innovative researches are focused not only on optimizing the extraction and composition performance; rather, they use variables that measure bioactivity $[20,21]$.

The aim of this study was to optimize the extraction conditions for the recovery of phytochemicals from L. citriodora leaves and H. sabdariffa calyces, considering their total polar content and antioxidant capacity, by different methods in order to obtain ingredients with high antioxidant potential. In addition, the optimization of extraction processes by MAE and PLE was compared, considering, on one hand, the total content of polar compounds measured by high-performance liquid chromatography coupled to mass spectrometry (HPLC-ESI-TOF/MS) and, on the other hand, the bioactivity measured by different in vitro tests.

The optimization was carried out using an RSM based on Central Composite Design $2^{3}$ (CCD) model with 16 experiments including center and star points. The independent variables were temperature, extraction time and percentage of solvent (ethanol and water) and the response variables were total phenolic compounds measured by HPLC-ESI-TOF/MS and by Folin-Ciocalteu assay, antioxidant capacity evaluated by Ferric Reducing Antioxidant Power (FRAP) assay and Trolox Equivalent Antioxidant Capacity (TEAC).

\section{Materials and Methods}

\subsection{Chemicals and Reagents}

For extractions and solutions, ultrapure water was obtained with a Milli-Q system Millipore (Bedford, MA, USA) and absolute ethanol was purchased from VWR chemicals (Radnor, PA, USA). To measure the antioxidant capacity and total phenolic content, the following reagents were provided from the indicated suppliers: ABTS (2,2'-azinobis (3-ethylbenzothiazoline-6-sulphonate)), ferric sulfate, Folin-Ciocalteu reagent, potassium persulfate, TPTZ (2,4,6-Tris(2-pyridyl)-s-triazine), Trolox (6-hydroxy-2,5,7,8-tetramethylchroman-2-carboxylic acid) from Sigma-Aldrich (St. Louis, MO, USA). 
Acetic acid and methanol were purchased from Fluka (Sigma-Aldrich, Steinheim, Germany) and Lab-Scan (Gliwice, Poland), respectively, whereas gallic acid, sodium acetate, ferric chloride, hydrochloric acid, trihydrated sodium acetate and sodium carbonate were obtained from Panreac (Barcelona, Spain).

The analytical procedures were performed using LC-MS grade methanol and acetic acid, which were purchased from Fisher Chemicals (Waltham, MA, USA) and Sigma-Aldrich (Steinheim, Germany), respectively.

\subsection{Sample Preparation}

H. sabdariffa calyces and L. citriodora leaves were provided by Monteloeder (Alicante, Spain) whose calyces and leaves were milled using an ultra-centrifugal mill ZM 200 (Retsch GmbH, Haan, Germany). The resulting powder was stored in darkness and kept at room temperature until extraction.

\subsection{Extraction of Phenolic Compounds and Other Polar Compounds from H. sabdariffa Calyces}

MAE was carried out in according to the methodology used by Pimentel-Moral et al. [17] in a modular microwave extraction system Multiwave 3000 SOLV (Anton Paar GmbH, Graz, Austria). Briefly, $3 \mathrm{~g}$ of $\mathrm{H}$. sabdariffa dried calyces were added into the vessels together with $30 \mathrm{~mL}$ of water-ethanol mixtures. After cooling, samples were centrifuged at 17,000 $\mathrm{g}$ for $15 \mathrm{~min}$ in a centrifuge (Sorvall ST 16 R, Thermo Scientific, Leicestershire, UK) and the supernatants were dried under vacuum in a Savant ${ }^{\mathrm{TM}}$ SpeedVac Concentrator SC250 EXP (Thermo analysis Scientific, Sunnyvale, CA, USA). The extracts were stored at $-20{ }^{\circ} \mathrm{C}$ until further use. Prior to use, the dry extracts were reconstituted in the same extraction solvent mixture to a concentration of $10 \mathrm{mg} / \mathrm{mL}$ and filtered with single-use syringe filters (0.20 $\mu \mathrm{m}$ pore size).

\subsection{Extraction of Phenolic Compounds and Other Polar Compounds from L. citriodra Leaves}

PLE was run according to the methodology used by Leyva-Jiménez et al. [14] with a pressurized liquid extractor (ASE 350 system, Dionex, Sunnyvale, CA, USA). Briefly, for each extraction, $5 \mathrm{~g}$ of sample were mixed with $10 \mathrm{~g}$ of sea sand and loaded onto $33 \mathrm{~mL}$ stainless-steel extraction cells. After extraction, the extracts obtained were ice-cold to achieve a temperature of $20-25^{\circ} \mathrm{C}$ and centrifuged at $17,000 \times g$ for $15 \mathrm{~min}$ in a centrifuge (Sorvall ST 16R, Thermo Scientific, Leicestershire, UK). The supernatants were dried under vacuum in a Savant ${ }^{\mathrm{TM}}$ SpeedVac Concentrator SC250 EXP (Thermo analysis Scientific, Sunnyvale, CA, USA) and they were stored at $-20{ }^{\circ} \mathrm{C}$ until further use. Prior to use, the dry extracts were reconstituted with the same extraction solvent mixture to a concentration of $10 \mathrm{mg} / \mathrm{mL}$ and filtered with single-use syringe filters $(0.20 \mu \mathrm{m}$ pore size $)$.

\subsection{Experimental Design}

For both MAE and PLE extractions, the experimental conditions were defined according to an RSM using a CCD $2^{3}$ model with axial and center points. RSM was designed with Statgraphics Centurion XVI software (Statpoint Technologies (Warrenton, VA, USA). In the MAE of $H$. sabdariffa calyces, the independent variables were temperature $\left(50,100,150{ }^{\circ} \mathrm{C}\right)$, extraction time $(5,12.5,20 \mathrm{~min})$ and percentage of ethanol in the hydro-alcoholic mixture $(15 \%, 45 \%, 75 \%)$. Conditions were selected based on Pimentel-Moral et al. [17]. A total of 16 experimental conditions were obtained (Table S1) that were carried out in a randomized order.

In the PLE of L. citriodora leaves, the independent variables were temperature $\left(40,110,180^{\circ} \mathrm{C}\right)$, extraction time $(5,12.5,20 \mathrm{~min})$ and percentage of ethanol in the hydro-alcoholic $(15 \%, 50 \%, 85 \%)$. Conditions were selected based on Leyva-Jiménez et al. [14]. A total of 16 experimental conditions were obtained (Table S2) that were carried out in a randomized order.

Total polar compounds were quantified by HPLC-ESI-TOF/MS according to Pimentel-Moral et al. in H. sabdariffa extracts and adapted from Leyva-Jiménez et al. in the case of L. citriodora extracts; total phenolic compounds were measured by Folin-Ciocalteu assay, whereas antioxidant capacity was evaluated using FRAP and by TEAC. The results of each assay were chosen as response variables in 
both models. The following second-order polynomial model was used to fit the experimental data (Equation (1)):

$$
Y=\beta_{0}+\sum_{i=1}^{k} \beta_{i} X_{i}+\sum_{i=1}^{k} \beta_{i i} X_{i}^{2}+\sum_{i=1}^{k} \sum_{j=i+1}^{k} \beta_{i j} X_{i} X_{j}
$$

$Y$ represents the predicted response; $\beta_{0}$ is a constant coefficient that fixes the response at the central point of the experiments, and $\beta_{i}, \beta_{i i}$ and $\beta_{i j}$ are the regression coefficients of the linear, quadratic and interaction terms, respectively; $X_{i}$ and $X_{j}$ represent the value of the independent variables. The parameters were considered to evaluate the model adequacy: regression coefficient $\left(R^{2}\right)$, coefficient of variation $(\mathrm{CV})$ and model value.

\subsection{Total Phenolic Content by Folin Ciocalteu}

The total phenolic content (TPC) was measured by Folin-Ciocalteu method with some modifications [22]. L. citriodora and H. sabdariffa extracts were dissolved in ethanol-water (1:1) (different concentrations were tested). The absorbance measurement was carried out on a Synergy Mx Monochromator-Based Multi-Mode Micro plate reader (Bio-Tek Instruments Inc., Winooski, VT, USA) using 96-well polystyrene microplates. Phenol content was calculated based on the calibration curves of Gallic Acid and expressed as mg Gallic acid equivalents/g of dry extract. The experiments were made in triplicate.

\subsection{Antioxidant Activity Assays}

The antioxidant activity of L. citriodora and H. sabdariffa extracts were evaluate using two methods based on single-electron transfer (SET): FRAP and TEAC assays. In both, fluorescence was measured with the same equipment mentioned above.

\subsubsection{Ferric Reducing Antioxidant Power}

The FRAP assay was carried out following the method described by Benzie and Strain [23]. FRAP values were calculated measuring the absorbance at $593 \mathrm{~nm}$ in a microplate reader. A standard curve of $\mathrm{FeSO}_{4} \cdot 7 \mathrm{H}_{2} \mathrm{O}$ was assessed and results were expressed as $\mathrm{mmol} \mathrm{FeSO}_{4}$ equivalents/g of dry extract. The experiments were made in triplicate.

\subsubsection{Trolox Equivalent Antioxidant Capacity}

The TEAC assay was originally described by Miller et al. and was performed with some modifications according to Cádiz-Gurrea et al. [24,25]. TEAC values were calculated using Trolox as standard and reading absorbance at $734 \mathrm{~nm}$ in a microplate reader. The results were expressed in mmol Trolox equivalents/g of dry extract. The experiments were made in triplicate.

\subsection{HPLC-MS Analysis}

The qualitative characterization of $H$. sabdariffa and L. citriodora extracts was carried out using a RRPC 1200 series (Agilent Technologies, Palo Alto, CA, USA) following the methods reported by Pimentel-Moral et al. [17] and Leyva-Jiménez et al. [14], respectively, in order to ensure the reproducibility.

\section{Results and Discussion}

\subsection{Total Polar Compounds, Total Phenolic Contents and Antioxidant Activities of H. sabdariffa by Microwave-Assisted Extraction (MAE)}

The analysis of the beneficial properties of plant extracts can be approached from different points of view. For instance, attention can be focused on the composition of bioactive compounds as evaluating a wide group of compounds as polar compounds or more specific as phenolic compounds, since these are responsible for the vast majority of beneficial properties reported. On the other hand, the studies can 
be focus on the bioactivity, such as antioxidant capacity of the extracts that may be mostly associated with the phenolic profile of this type of polar extractions, but taking into account the positive and negative synergies that have influence in the antioxidant properties. It is necessary to consider that the results of each applied assay to evaluate this bioactivity may change according to the chosen technique. For these reasons, this work pretends to approach the relationship between phytochemicals contained in the extracts and their antioxidant activity in order to establish optimal conditions for the obtainment of functional ingredients.

In this sense, Table 1 shows the results of the total content of polar compounds adapted from Pimentel-Moral et al. [17], the TPC measured by the Folin-Ciocalteu and the antioxidant activity obtained by the FRAP and TEAC assays for each of the experimental conditions of MAE extraction of $H$. sabdariffa calyces. In this case, the extraction yield has been used to ensure the reproducibility of the extractions with those made by Pimentel-Moral et al. [17], noting that there were no non-significant differences (data not shown). Table 2 shows the major compounds tentatively identified in H. sabdariffa calyces by HPLC-ESI-TOF-MS following the method reported by Pimentel-Moral et al. [17].

As can be seen in Table 1, there is no condition in which all the response variables reach the maximum; however, when attention is paid to the condition with the lowest value for the response variables, it is observed that condition 10 has the lowest value for the four response variables. These results indicate that the composition of the H. sabdariffa extracts have an important influence on the antioxidant activity.

The results of Total Polar Compounds variable ranged from $201.466 \pm 5.356 \mathrm{mg}$ of total polar compounds/g of dry extract (run 11) to $50.027 \pm 1.107 \mathrm{mg}$ total polar compounds/g of dry extract (run 10); the extraction conditions under these conditions were $164{ }^{\circ} \mathrm{C}, 12.5 \mathrm{~min}$ and $45 \%$ ethanol, and $150{ }^{\circ} \mathrm{C}, 20 \mathrm{~min}$ and $15 \%$ ethanol, respectively. For Folin-Ciocalteu response, the condition with the highest value was run 8 with $78.679 \pm 2.591 \mathrm{mg}$ Gallic acid equivalents/g of dry extract under the conditions $150{ }^{\circ} \mathrm{C}, 5 \mathrm{~min}, 75 \%$ ethanol; for FRAP, conditions 5 and 15 with $0.995 \pm 0.034$ and $0.995 \pm 0.018 \mathrm{mmol} \mathrm{FeSO}_{4}$ equivalents/g of dry extract under $100^{\circ} \mathrm{C}$, and $45 \%$ ethanol, and for TEAC, condition 11 gave $0.285 \pm 0.010 \mathrm{mmol}$ Trolox equivalents/g of dry extract. The results achieved by condition 11 offered higher values for total polar compounds as well as TEAC assay, which may highlight the synergic effect of polar compounds in the antioxidant activity. Some examples of this synergistic effect on antioxidant activity between phenolic compounds was evaluated by Skroza et al., in which they observed that in different tests to measure antioxidant capacity there was a synergistic effect between resveratrol and other phenols such as catechin and caffeic acid [26]. Also in this sense, in the case of the TEAC assay, Sanchez-Marzo et al. tested different fractions of compounds present in L. citriodora, checking the fraction with a combination of Apigenin-7-diglucuronide, Verbascoside, Isoverbascoside and Forsythoside A presented higher values in both FRAP and TEAC, compared to other fractions with different combinations or with isolated compounds. Thus, this demonstrates the synergistic effect of the compounds present in this fraction [27]. As mentioned above, condition 10 gave the lowest values for all responses evaluated. 
Table 1. Results of the response variables Total Polar Compounds, TPC, FRAP and TEAC for each condition in H. sabdariffa MAE extracts.

\begin{tabular}{ccccc}
\hline Experimental Design Condition & Total Polar Compounds ${ }^{\mathbf{a}, \mathbf{1}}$ & TPC $^{\mathbf{b}}$ & FRAP $^{\mathbf{c}}$ & TEAC $^{\mathbf{d}}$ \\
\hline 1 & $92.514 \pm 4.727$ & $41.114 \pm 1.832$ & $0.935 \pm 0.036$ & $0.254 \pm 0.009$ \\
2 & $76.171 \pm 1.501$ & $57.953 \pm 2.591$ & $0.959 \pm 0.044$ & $0.260 \pm 0.002$ \\
3 & $56.469 \pm 3.547$ & $39.822 \pm 0.006$ & $0.767 \pm 0.024$ & $0.213 \pm 0.004$ \\
4 & $96.682 \pm 1.676$ & $72.634 \pm 5.393$ & $0.781 \pm 0.062$ & $0.268 \pm 0.009$ \\
5 & $83.807 \pm 1.365$ & $52.772 \pm 4.487$ & $0.995 \pm 0.034$ & $0.284 \pm 0.008$ \\
6 & $84.987 \pm 1.934$ & $43.273 \pm 3.957$ & $0.757 \pm 0.043$ & $0.216 \pm 0.004$ \\
7 & $58.774 \pm 1.767$ & $25.570 \pm 1.832$ & $0.651 \pm 0.027$ & $0.182 \pm 0.005$ \\
8 & $87.112 \pm 4.804$ & $78.679 \pm 2.591$ & $0.711 \pm 0.054$ & $0.228 \pm 0.013$ \\
9 & $81.339 \pm 1.511$ & $45.864 \pm 1.496$ & $0.799 \pm 0.016$ & $0.233 \pm 0.003$ \\
10 & $50.027 \pm 1.107$ & $26.002 \pm 1.496$ & $0.524 \pm 0.011$ & $0.133 \pm 0.006$ \\
11 & $201.466 \pm 5.356$ & $61.408 \pm 2.991$ & $0.819 \pm 0.024$ & $0.285 \pm 0.010$ \\
12 & $53.368 \pm 1.331$ & $40.682 \pm 1.496$ & $0.537 \pm 0.024$ & $0.166 \pm 0.003$ \\
13 & $56.314 \pm 0.951$ & $44.136 \pm 3.957$ & $0.607 \pm 0.024$ & $0.190 \pm 0.001$ \\
14 & $77.399 \pm 0.682$ & $74.361 \pm 5.983$ & $0.968 \pm 0.022$ & $0.280 \pm 0.006$ \\
15 & $71.110 \pm 0.937$ & $54.499 \pm 2.991$ & $0.995 \pm 0.018$ & $0.261 \pm 0.001$ \\
16 & $69.784 \pm 1.262$ & $42.407 \pm 0.003$ & $0.903 \pm 0.043$ & $0.232 \pm 0.003$ \\
\hline
\end{tabular}

${ }^{\mathrm{a}}$ Expressed in mg total polar compounds/g of dry extract; ${ }^{\mathrm{b}}$ Expressed in $\mathrm{mg}$ Gallic acid equivalents/g of dry extract;

${ }^{\mathrm{c}}$ Expressed in $\mathrm{mmol} \mathrm{FeSO}_{4}$ equivalents/g of dry extract; ${ }^{\mathrm{d}}$ Expressed in mmol Trolox equivalents/g of dry extract;

${ }^{1}$ Adapted from Pimentel Moral et al. [17]; TPC: Folin-Ciocalteu; FRAP: Ferric Reducing Antioxidant Power; TEAC:

Trolox Equivalent Antioxidant Capacity.

Table 2. Major compounds tentatively identified in H. sabdariffa calyces by high-performance liquid chromatography coupled to mass spectrometry (HPLC-ESI-TOF-MS).

\begin{tabular}{ccc}
\hline Molecular Formula & $m / z$ Calculated & Compound \\
\hline $\mathrm{C}_{6} \mathrm{H}_{8} \mathrm{O}_{8}$ & 207.0146 & Hydroxicitric acid \\
$\mathrm{C}_{6} \mathrm{H}_{6} \mathrm{O}_{7}$ & 189.0041 & Hibiscus acid \\
$\mathrm{C}_{8} \mathrm{H}_{12} \mathrm{O}_{8}$ & 235.0459 & Hibiscus acid hydroxyethylester \\
$\mathrm{C}_{8} \mathrm{H}_{10} \mathrm{O}_{7}$ & 217.0354 & Hibiscus acid dimethylester \\
$\mathrm{C}_{16} \mathrm{H}_{18} \mathrm{O}_{9}$ & 353.0878 & Neochlorogenic acid \\
$\mathrm{C}_{16} \mathrm{H}_{18} \mathrm{O}_{9}$ & 353.0878 & Chlorogenic acid \\
$\mathrm{C}_{16} \mathrm{H}_{18} \mathrm{O}_{9}$ & 353.0878 & Cryptochlorogenic acid \\
$\mathrm{C}_{15} \mathrm{H}_{12} \mathrm{O}_{9}$ & 335.0409 & Methyl digallate \\
$\mathrm{C}_{16} \mathrm{H}_{18} \mathrm{O}_{8}$ & 337.0929 & Coumaroilquinic acid \\
$\mathrm{C}_{16} \mathrm{H}_{20} \mathrm{O}_{10}$ & 371.0984 & Dihydroferulic acid-4-O-glucuronide \\
$\mathrm{C}_{26} \mathrm{H}_{28} \mathrm{O}_{16}$ & 595.1305 & Quercetin-3-sambubioside \\
$\mathrm{C}_{17} \mathrm{H}_{30} \mathrm{O}_{16}$ & 489.1461 & Quercetin-3-rutinoside \\
$\mathrm{C}_{16} \mathrm{H}_{16} \mathrm{O}_{8}$ & 335.0772 & 5-O-caffeoylshikimic acid \\
$\mathrm{C}_{21} \mathrm{H}_{20} \mathrm{O}_{12}$ & 463.0882 & Quercetin-3-glucoside \\
$\mathrm{C}_{18} \mathrm{H}_{22} \mathrm{O}_{9}$ & 381.1191 & Ethylchlorogenate \\
$\mathrm{C}_{18} \mathrm{H}_{22} \mathrm{O}_{9}$ & 381.1191 & Ethylchlorogenate isomer II \\
$\mathrm{C}_{16} \mathrm{H}_{16} \mathrm{O}_{7}$ & 319.0823 & Methylepigallocatechin \\
$\mathrm{C}_{15} \mathrm{H}_{10} \mathrm{O}_{8}$ & 317.0303 & Myricetin \\
$\mathrm{C}_{18} \mathrm{H}_{19} \mathrm{NO}_{4}$ & 312.1241 & $N$-feruloyltiramine \\
$\mathrm{C}_{15} \mathrm{H}_{10} \mathrm{O}_{7}$ & 301.0354 & Quercetin \\
$\mathrm{C}_{15} \mathrm{H}_{10} \mathrm{O}_{6}$ & 285.0405 & Kaempferol \\
\hline
\end{tabular}

In spite of run 11 revealing the highest total polar compounds in the evaluated design, the value reached in Folin-Ciocalteu by condition 8 was the highest value. This unexpected value may be due to the fact that Folin-Ciocalteu reagent not only reacts with phenolic compounds, which are included within polar compounds, but also reacts with others such as proteins, thiols, many vitamin derivatives and inorganic ions $\mathrm{Fe}^{2+}, \mathrm{Mn}^{2+}, \mathrm{I}^{-}$and $\mathrm{SO}_{3}{ }^{2-}$ [28]. According to these results, higher percentage of ethanol from $45 \%$ (run 11 ) to $75 \%$ (run 8 ) allowed greater extraction of pigments such as chlorophyll, carotenoids and xanthophylls [29], which can be conjugated with proteins and have some of the aforementioned ions in their structure, reacting with the Folin-Ciocalteu reagent. Additionally, 
the lowest and least maintained temperature over time of condition 8 compared to 11 prevents the degradation of certain proteins and vitamins, explaining the high value for Folin-Ciocalteu of condition 8 [30].

Considering the response variables to evaluate the antioxidant capacity, condition 11 showed the highest TEAC value and the greatest total polar compounds. Moreover, the values of organic acids, phenolic acids, flavonoids and anthocyanins showed the highest, so that in relation to TEAC the greatest concentration of compounds is directly related to the highest antioxidant activity. However, in the case of FRAP, the results differ, since there were two conditions that revealed greater values than conditions 11, 5 and 15 that were performed with $45 \%$ of ethanol. Moreover, the temperature had an important relevance since, while in condition 11 the extraction temperature was $164{ }^{\circ} \mathrm{C}$, in 5 and 15 it was $100^{\circ} \mathrm{C}$. These results may be occasioned by the thermal degradation of compounds like flavonoids. Quercetin and its derivatives are flavonoids that were found in $H$. sabdariffa extracts, and their abilities to reduce iron in the FRAP test compared to other flavonoids have been reported [31]. According to Pimentel-Moral et al., in conditions 5 and 15, the content of quercetin-3-rutinoside was greater than condition 11, which could explain the higher value in FRAP of these two conditions [17].

\subsection{Total Polar Compounds, Total Phenolic Contents and In Vitro Antioxidant Activities of L. citriodora Pressurized Fluid Extraction (PLE)}

Table 3 shows the results attained after evaluating the PLE L. citriodora extracts, where the results of total content of polar compounds were adapted from Leyva-Jiménez et al. [14], whereas the TPC was measured applying the Folin-Ciocalteu assay and the antioxidant activity performing FRAP and TEAC assays. In the same way that was mentioned previously for $H$. sabdariffa, the extraction yield has been the parameter used to compare the extractions carried out in this study with those made by Leyva-Jiménez et al. [14], noting that there were no non-significant differences (data not shown). Table 4 shows the major compounds tentatively identified in L. citriodora leaves by HPLC-ESI-TOF-MS following the method described by Leyva-Jiménez et al. [14].

Table 3. Results of the response variables for each condition in L. citriodora. Response variables: Total Polar Compounds, TPC, FRAP and TEAC.

\begin{tabular}{ccccc}
\hline Experimental Design Condition & Total Polar Compounds ${ }^{\mathbf{a}, 1}$ & TPC $^{\mathbf{b}}$ & FRAP $^{\mathbf{c}}$ & TEAC $^{\mathbf{d}}$ \\
\hline 1 & $304.734 \pm 21.965$ & $181.442 \pm 9.098$ & $1.720 \pm 0.080$ & $0.64 \pm 0.028$ \\
2 & $369.613 \pm 24.362$ & $253.117 \pm 1.496$ & $2.402 \pm 0.131$ & $0.553 \pm 0.054$ \\
3 & $213.096 \pm 13.480$ & $168.489 \pm 5.393$ & $2.095 \pm 0.048$ & $0.580 \pm 0.068$ \\
4 & $220.119 \pm 11.880$ & $195.259 \pm 4.487$ & $1.997 \pm 0.063$ & $0.524 \pm 0.005$ \\
5 & $199.962 \pm 9.621$ & $159.853 \pm 10.786$ & $1.745 \pm 0.098$ & $0.653 \pm 0.053$ \\
6 & $268.933 \pm 17.803$ & $235.846 \pm 7.915$ & $1.802 \pm 0.058$ & $0.553 \pm 0.019$ \\
7 & $228.959 \pm 17.110$ & $197.850 \pm 2.591$ & $1.875 \pm 0.120$ & $0.438 \pm 0.022$ \\
8 & $221.533 \pm 12.409$ & $227.211 \pm 12.779$ & $2.313 \pm 0.055$ & $0.574 \pm 0.029$ \\
9 & $141.768 \pm 9.291$ & $145.173 \pm 7.479$ & $1.685 \pm 0.153$ & $0.555 \pm 0.049$ \\
10 & $252.724 \pm 10.062$ & $219.439 \pm 3.957$ & $1.615 \pm 0.125$ & $0.553 \pm 0.031$ \\
11 & $329.635 \pm 20.249$ & $193.532 \pm 3.957$ & $2.252 \pm 0.085$ & $0.532 \pm 0.085$ \\
12 & $226.461 \pm 8.706$ & $189.214 \pm 1.496$ & $1.728 \pm 0.111$ & $0.420 \pm 0.006$ \\
13 & $155.232 \pm 28.764$ & $211.667 \pm 1.496$ & $1.877 \pm 0.069$ & $0.493 \pm 0.024$ \\
14 & $282.828 \pm 16.771$ & $242.755 \pm 6.520$ & $2.318 \pm 0.031$ & $0.665 \pm 0.002$ \\
15 & $125.646 \pm 5.013$ & $185.760 \pm 7.915$ & $1.230 \pm 0.048$ & $0.453 \pm 0.022$ \\
16 & $96.830 \pm 4.096$ & $163.307 \pm 1.496$ & $1.590 \pm 0.056$ & $0.558 \pm 0.088$ \\
\hline
\end{tabular}

\footnotetext{
${ }^{a}$ Expressed in $\mathrm{mg}$ total polar compounds/g of dry extract; ${ }^{\mathrm{b}}$ Expressed in $\mathrm{mg}$ Gallic acid equivalents/g of dry extract;

${ }^{c}$ Expressed in $\mathrm{mmol} \mathrm{FeSO}_{4}$ equivalents/g of dry extract; ${ }^{\mathrm{d}}$ Expressed in mmol Trolox equivalents/g of dry extract;

1 Adapted from Leyva-Jiménez et al. [14] with some modifications; TPC: Folin-Ciocalteu; FRAP: Ferric Reducing

Antioxidant Power; TEAC: Trolox Equivalent Antioxidant Capacity.
} 
Table 4. Major compounds tentatively identified in L. citriodora leaves by high-performance liquid chromatography coupled to mass spectrometry (HPLC-ESI-TOF-MS).

\begin{tabular}{ccc}
\hline Molecular Formula & $m / z$ Calculated & Compound \\
\hline $\mathrm{C}_{6} \mathrm{H}_{11} \mathrm{O}_{7}$ & 195.0510 & Gluconic acid \\
$\mathrm{C}_{16} \mathrm{H}_{21} \mathrm{O}_{10}$ & 373.1140 & Gardoside \\
$\mathrm{C}_{16} \mathrm{H}_{19} \mathrm{O}_{11}$ & 387.0933 & Ixoside \\
$\mathrm{C}_{20} \mathrm{H}_{29} \mathrm{O}_{12}$ & 461.1664 & Verbasoside \\
$\mathrm{C}_{21} \mathrm{H}_{27} \mathrm{O}_{13}$ & 487.1457 & Cistanoside $\mathrm{F}$ \\
$\mathrm{C}_{16} \mathrm{H}_{21} \mathrm{O}_{11}$ & 389.1089 & Theveside \\
$\mathrm{C}_{18} \mathrm{H}_{27} \mathrm{O}_{9}$ & 387.1661 & Tuberonic acid glucoside \\
$\mathrm{C}_{29} \mathrm{H}_{37} \mathrm{O}_{16}$ & 641.2087 & $\beta$ Hydroxyverbascoside derivative \\
$\mathrm{C}_{29} \mathrm{H}_{37} \mathrm{O}_{16}$ & 641.2087 & $\beta$ Hydroxyisoverbascoside derivative \\
$\mathrm{C}_{29} \mathrm{H}_{35} \mathrm{O}_{16}$ & 639.1931 & $\beta$ Hydroxyverbascoside \\
$\mathrm{C}_{27} \mathrm{H}_{25} \mathrm{O}_{18}$ & 637.1140 & Luteolin-7-diglucoronide \\
$\mathrm{C}_{29} \mathrm{H}_{35} \mathrm{O}_{16}$ & 639.1931 & $\beta$ Hydroxyisoverbascoside \\
$\mathrm{C}_{27} \mathrm{H}_{25} \mathrm{O}_{17}$ & 621.1097 & Apigenin-7-diglucoronide \\
$\mathrm{C}_{25} \mathrm{H}_{29} \mathrm{O}_{13}$ & 537.1614 & Lippioside I \\
$\mathrm{C}_{30} \mathrm{H}_{37} \mathrm{O}_{16}$ & 653.2087 & Campneoside I \\
$\mathrm{C}_{28} \mathrm{H}_{27} \mathrm{O}_{18}$ & 651.1355 & Chrysoeriol-7-diglucuronide \\
$\mathrm{C}_{29} \mathrm{H}_{35} \mathrm{O}_{15}$ & 623.1981 & Verbascoside \\
$\mathrm{C}_{26} \mathrm{H}_{33} \mathrm{O}_{11}$ & 521.2028 & Lariciresinol glucopyranoside \\
$\mathrm{C}_{29} \mathrm{H}_{35} \mathrm{O}_{15}$ & 623.1981 & Isoverbascoside \\
$\mathrm{C}_{29} \mathrm{H}_{35} \mathrm{O}_{15}$ & 623.1981 & Forsythoside A \\
$\mathrm{C}_{25} \mathrm{H}_{29} \mathrm{O}_{12}$ & 521.1664 & Hydroxycampsiside \\
$\mathrm{C}_{26} \mathrm{H}_{31} \mathrm{O}_{13}$ & 551.1770 & Durantoside I \\
$\mathrm{C}_{30} \mathrm{H}_{37} \mathrm{O}_{15}$ & 637.2138 & Leucoseptoside A \\
$\mathrm{C}_{28} \mathrm{H}_{27} \mathrm{O}_{17}$ & 635.1254 & Acacetin-7-diglucoronide \\
$\mathrm{C}_{31} \mathrm{H}_{39} \mathrm{O}_{15}$ & 651.2294 & Martynoside \\
$\mathrm{C}_{29} \mathrm{H}_{35} \mathrm{O}_{13}$ & 591.2083 & Osmanthuside B \\
$\mathrm{C}_{16} \mathrm{H}_{11} \mathrm{O}_{6}$ & 299.0561 & Dimethyl kaempferol \\
$\mathrm{C}_{17} \mathrm{H}_{13} \mathrm{O}_{7}$ & 329.0667 & Dimethyl quercetin \\
\hline
\end{tabular}

Concerning the FRAP response variable, the condition with the highest value coincided with Total Polar Compounds and Folin-Ciocalteu, pointing out a positive relationship between the amount of phenolic compounds and the ability to reduce the iron ion through the transfer of electrons. Furthermore, in accordance with Leyva-Jiménez et al., run 2 showed the highest amount of verbascoside, which may explain the antioxidant results of this run. This could be corroborated by various studies that show the positive relationship between the amount of verbascoside in the matrix and the highest capacity in the FRAP test [27,32].

Nevertheless, for the TEAC response variable, the condition run 14 exhibited the highest value. These results may be explained due to synergistic relationships between the phytochemicals contained in this extract and showing that the amounts of polar/phenolic compounds were not related with reduction of the ABTS cation radical. There are studies that show that there is not always a direct relationship between the relative concentration of a compound or certain compounds with the bioactivity, but in many situations, synergistic or antagonist relationships are determinant to the bioactive potential of the extracts obtained from plants [33].

Lastly, it is necessary to note that the minimum value of all responses evaluated was achieved after applying higher temperatures (above $180^{\circ} \mathrm{C}$ ). This may be due to the fact that there were compounds that are thermolabile at high temperatures and therefore could be degraded [12]. 


\subsection{RSM Analysis of H. sabdariffa Calyces MAE and L. citriodora Leaves PLE Conditions}

\subsubsection{Model Fitting Parameters}

To maximize the response variables for each model, an analysis of variance (ANOVA) was carried out for each response to discern the adjustment of the results and optimize the statistical model. To achieve this goal, several fitting parameters were used, which were evaluated to determine the adequacy of the model. The results were fitted to a quadratic polynomial model (Equations (S1) and (S2)) and regression coefficients were generated using the least squares method (MLS). The first parameter to evaluate the adequacy of the model was the regression coefficient $\left(R^{2}\right)$, in which values above $70 \%$ are considered acceptable [34]. This value explains the variability of the data which may be explained with the proposed model. The coefficient of variation (CV) was also used, a lower value $(<10 \%)$ indicates a good reproducibility of the investigated systems and a value between $11 \%$ and $20 \%$, indicates an acceptable variation; hence, $\mathrm{CV}$ shows the dispersion of data and a small value indicated high reproducibility between the predicted and experimental values [35]. Finally, the adequacy of the model (model value) was also used as approach to discern the good choice of the design. Table 5 shows the parameters mentioned to validate the model in H. sabdariffa and Table 6 the results of the ANOVA for this matrix. Table 7 shows the parameters mentioned to validate the model in L. citriodora and Table 8 the results of the ANOVA for this matrix.

\subsubsection{H. sabdariffa Calyces MAE Optimization}

Regarding the evaluated fitting parameters (Table 5), Folin-Ciocalteu, FRAP and TEAC response variables showed $R^{2}$ values greater than $70 \%$ (93.7\%, 78.4\% and $74.0 \%$, respectively), hence revealing a good explanation of the obtained results. Moreover, the adequacy of the model of each response gave significant values $(p \leq 0.05)$ demonstrating a good adequacy, and the CV was below 20 and close to 10 , revealing an acceptable reproducibility of the results. Nevertheless, Total Polar Compound did not present a good approximation to the experimental conditions since neither the result of $R^{2}$ model value nor $\mathrm{CV}$ was acceptable.

Considering the effects of the independent variables, it is noteworthy that the \% ethanol had a significant influence for the Folin-Ciocalteu, FRAP and TEAC response. Furthermore, the optimization of these responses (Table 6) was achieved when the ethanol used was between $67 \%$ and $83 \%$. This could be explained because water, despite having higher dielectric constant, has a dissipation factor significantly lower than ethanol. In this sense, a high dissipation factor improves the passage of microwave energy through the solvent and the dissipation of microwave energy into heat, as is the case with ethanol [36]. Despite this characteristic, its ability to absorb energy is low. This causes less heat transfer from the solvent to the sample [37], requiring water in the solvent mixture to improve this situation. With these observations, a higher extraction yield of phenolic compounds were obtaining using $80 \%$ of ethanol in the mixture, comparing with water and other mixtures of ethanol-water and methanol-water, which coincide with the results obtained in the present study [36].

Furthermore, Total Polar Compounds and Folin-Ciocalteu showed the optimal temperatures at $164^{\circ} \mathrm{C}$ and the extraction time was 13 and $3 \mathrm{~min}$, respectively. Nevertheless, the optimal temperature for FRAP was $62^{\circ} \mathrm{C}$, while for TEAC it was $118^{\circ} \mathrm{C}$, and the optimal extraction time was 12 and 13 min, respectively. In these cases, the temperature necessary to obtain the optimal values of FRAP and TEAC enabled the extraction of thermo sensitive compounds as flavonoids. These flavonoids had a greater affinity for reducing iron in FRAP compared to the TEAC test based on the ABTS radical. These flavonoids had a higher affinity for reducing iron in FRAP compared to the ABTS radical-based TEAC test. This result may be explained considering the results attained by Bahorun et al. and Csepregi et al., which conducted FRAP and TEAC tests for different types of flavonoids. The results of their studied revealed that particularly quercetin and quercetin-3-O-rutinoside, very abundant in $H$. sabdariffa, had higher values for FRAP than for TEAC $[38,39]$. This can be explained since the 
presence an aliphatic substituent and a double bond on a catechol ring is positively associated with the iron ion reduction [40].

Table 5. Analysis of variance (ANOVA) of the regression models in H. sabdariffa. Response variables: Total Polar Compounds, TPC, FRAP and TEAC.

\begin{tabular}{ccccc}
\hline \multirow{2}{*}{ Source } & Total Polar Compounds & TPC & FRAP & TEAC \\
\cline { 2 - 5 } & $p$ Value & $p$ Value & $p$ Value & $p$ Value \\
\hline $\mathrm{X}_{1}$ : Temperature & 0.1796 & $0.0306^{\mathrm{a}}$ & $0.0798^{\mathrm{b}}$ & 0.6682 \\
$\mathrm{X}_{2}$ : Solvent & 0.2141 & $0.0003^{\mathrm{a}}$ & $0.0131^{\mathrm{a}}$ & $0.0118^{\mathrm{a}}$ \\
$\mathrm{X}_{3}:$ Time & 0.7662 & $0.0459^{\mathrm{a}}$ & 0.8848 & 0.9187 \\
$\mathrm{X}_{1} \mathrm{X}_{1}$ & 0.2567 & 0.6735 & 0.2945 & 0.6745 \\
$\mathrm{X}_{1} \mathrm{X}_{2}$ & 0.8091 & $0.0078^{\mathrm{a}}$ & 0.7945 & 0.4297 \\
$\mathrm{X}_{1} \mathrm{X}_{3}$ & 0.9191 & 0.9815 & 0.8184 & 0.9240 \\
$\mathrm{X}_{2} \mathrm{X}_{2}$ & 0.1766 & 0.9960 & 0.1263 & 0.1806 \\
$\mathrm{X}_{2} \mathrm{X}_{3}$ & 0.7743 & 0.8339 & 0.7707 & 0.6862 \\
$\mathrm{X}_{3} \mathrm{X}_{3}$ & 0.2769 & 0.9588 & 0.5047 & 0.4107 \\
Model value & 0.2631 & $0.0004^{\mathrm{a}}$ & $0.0101^{\mathrm{a}}$ & $0.0168^{\mathrm{a}}$ \\
\hline$R^{2}$ & 0.621 & 0.937 & 0.784 & 0.740 \\
$\mathrm{CV}$ & $<29.64$ & $<12.79$ & $<13.81$ & $<13.50$
\end{tabular}

$R^{2}=$ Quadratic correlation coefficient; CV = Coefficient of variation (\%); ${ }^{\text {a Significant }(p<0.050) ;{ }^{b} \text { Marginally }}$ significant $(p<0.100)$; TPC: Folin-Ciocalteu; FRAP: Ferric Reducing Antioxidant Power; TEAC: Trolox Equivalent Antioxidant Capacity.

Table 6. Values of the independent conditions to optimize the value of the response variables in H. sabdariffa. Response variables: Total Polar Compounds, TPC, FRAP and TEAC.

\begin{tabular}{ccccc}
\hline Source & Total Polar Compounds & TPC & FRAP & TEAC \\
\hline $\mathrm{X}_{1}$ : Temperature $^{\mathrm{a}}$ & 164.36 & 164.36 & 64.91 & 118.15 \\
$\mathrm{X}_{2}$ : Solvent $^{\mathrm{b}}$ & 57.33 & 83.62 & 67.08 & 72.56 \\
$\mathrm{X}_{3}$ : Time $^{\mathrm{c}}$ & 13.15 & 2.85 & 11.70 & 13.43 \\
Theoretical optimum value $^{\mathrm{N}}$ & $150.456^{\mathrm{d}}$ & $97.742 \mathrm{e}$ & $0.991^{\mathrm{f}}$ & $0.278 \mathrm{~g}$ \\
\hline
\end{tabular}

a Expressed in ${ }^{\circ} \mathrm{C} ;{ }^{b}$ Expressed in \% of ethanol; ${ }^{\mathrm{c}}$ Expressed in minutes; ${ }^{\mathrm{d}}$ Expressed in mg total polar compounds $/ \mathrm{g}$ of dry extract; ${ }^{\mathrm{e}}$ Expressed in $\mathrm{mg}$ Gallic acid equivalents/g of dry extract; ${ }^{\mathrm{f}}$ Expressed in mmol $\mathrm{FeSO}_{4}$ equivalents/g of dry extract; ${ }^{g}$ Expressed in mmol Trolox equivalents/g of dry extract; TPC: Folin-Ciocalteu; FRAP: Ferric Reducing Antioxidant Power; TEAC: Trolox Equivalent Antioxidant Capacity.

\subsubsection{L. citriodora Leaves PLE Model}

Table 7 shows variable responses for L. citriodora PLE extractions, which gave $R^{2}$ values above 70\%, reaching values near 100\% in Total Polar Compounds and Folin-Ciocalteu responses: $95.5 \%$ and $91.8 \%$, respectively. With regard to the adequacy of the model, it can be observed that for Total Polar Compounds and Folin-Ciocalteu it was significant $(p \leq 0.05)$, while for the variables FRAP and TEAC, it was marginally significant $(p \leq 0.1)$. Finally, when the third parameter was analyzed, the CV was observed to be below $20 \%$ in the four variables. After analyzing these parameters, it can be considered that this model provided a good approximation and explanation of the behavior of each variable evaluated. 
Table 7. Analysis of variance (ANOVA) of the regression models in L. citriodora. Response variables: Total Polar Compounds, TPC, FRAP and TEAC.

\begin{tabular}{|c|c|c|c|c|}
\hline \multirow{2}{*}{ Source } & Total Polar Compounds & TPC & FRAP & TEAC \\
\hline & $p$ Value & $p$ Value & $p$ Value & $p$ Value \\
\hline $\mathrm{X}_{1}$ : Temperature & $0.0001^{\mathrm{a}}$ & $0.0241^{\mathrm{a}}$ & $0.0412^{\mathrm{a}}$ & $0.0511^{b}$ \\
\hline $\mathrm{X}_{2}$ : Solvent & $0.0023^{a}$ & $0.0009^{a}$ & $0.0556^{b}$ & 0.1760 \\
\hline $\mathrm{X}_{3}:$ Time & 0.4840 & 0.9881 & 0.9649 & 0.4579 \\
\hline$X_{1} X_{1}$ & 0.1580 & 0.2192 & $0.0424^{\mathrm{a}}$ & 0.4636 \\
\hline$x_{1} x_{2}$ & 0.4944 & 0.7579 & 0.2290 & 0.9206 \\
\hline$x_{1} x_{3}$ & $0.0430^{\mathrm{a}}$ & $0.0980^{b}$ & 0.7339 & 0.5773 \\
\hline$X_{2} X_{2}$ & 0.2570 & 0.1079 & 0.1320 & $0.0869^{b}$ \\
\hline$x_{2} x_{3}$ & $0.0520^{\mathrm{b}}$ & 0.1040 & 0.8596 & 0.7657 \\
\hline$x_{3} x_{3}$ & 0.9450 & $0.0315^{\mathrm{a}}$ & 0.9599 & 0.2396 \\
\hline Model value & $0.0001^{a}$ & $0.0057^{a}$ & $0.0852^{b}$ & $0.0892^{b}$ \\
\hline$R^{2}$ & 0.955 & 0.918 & 0.800 & 0.725 \\
\hline $\mathrm{CV}$ & $<15.12$ & $<7.22$ & $<13.04$ & $<10.09$ \\
\hline
\end{tabular}

$R^{2}=$ Quadratic correlation coefficient; CV = Coefficient of variation (\%); ${ }^{\text {a }}$ Significant $(p<0.050)$; ${ }^{\mathrm{b}}$ Marginally significant $(p<0.100)$; TPC: Folin-Ciocalteu; FRAP: Ferric Reducing Antioxidant Power; TEAC: Trolox Equivalent Antioxidant Capacity.

Regarding the effect of the independent variables, it is noted that for all response variables evaluated, temperature had a significant effect $(p \leq 0.05)$, except for TEAC, which made it marginally significant $(p \leq 0.1)$. The negative influence of temperature is exposed in Table 8 , which confirms that the temperature necessary to optimize these variables was very low (from 20 to $25^{\circ} \mathrm{C}$ ), with the exception of the FRAP, which was moderate $\left(71^{\circ} \mathrm{C}\right)$. Ethanol percentage had a significant effect in Total Polar Compounds and Folin-Ciocalteu response variables, whereas for FRAP it was marginally significant. Table 8 demonstrates the positive significant effect exerted since for these three variables the optimal percentage was $95 \%$ ethanol. When the temperature and ethanol data are viewed together, it can be thought that the optimal low temperatures for extraction are combined with high percentages of ethanol to increase the solubility of the compounds. Both parameters, lower temperatures and higher concentrations of ethanol, may induce a better recovery of phytochemicals according to their polarity. Therefore, the compounds extracted under these conditions allowed greater reduction of iron ion in FRAP assay.

Conversely, TEAC presented an optimal percentage of ethanol that was very low (5\%) (Table 8 ). This may be because the catechol group present in some phenolic compounds has a higher solubility in water [41]. As mentioned above, the catechol group is of great importance in the FRAP assay, but also in the TEAC assay. This catechol group has shown to have a fundamental role in the antioxidant capacity measured by TEAC, so phenolic compounds with more than one catechol group such as verbascoside, isoverbascoside and forsythoside $\mathrm{A}$, which are very abundant in L. citriodora, will play a key role in the antioxidant capacity measured by TEAC [27]. Furthermore, another group present in L. citriodora, which are extracted in greater quantities with low percentages of ethanol [14], are the glucuronized flavonoids, in which the number of catechol groups is greater. 
Table 8. Values of the independent conditions to optimize the value of the response variables in L. citriodora. Response variables: Total Polar Compounds, TPC, FRAP and TEAC.

\begin{tabular}{ccccc}
\hline Source & Total Polar Compounds & TPC & FRAP & TEAC \\
\hline $\mathrm{X}_{1}$ : Temperature $^{\mathrm{a}}$ & 26.51 & 19.90 & 71.12 & 26.25 \\
$\mathrm{X}_{2}$ : Solvent $^{\mathrm{b}}$ & 95.05 & 95.05 & 95.05 & 4.95 \\
$\mathrm{X}_{3}$ : Time $^{\mathrm{c}}$ & 2.85 & 6.93 & 22.05 & 11.47 \\
Theoretical optimum value $^{\mathrm{C}}$ & $374.344^{\mathrm{d}}$ & $252.952^{\mathrm{e}}$ & $2.541^{\mathrm{f}}$ & $0.696^{\mathrm{g}}$ \\
\hline
\end{tabular}

${ }^{\text {a }}$ Expressed in ${ }^{\circ} \mathrm{C} ;{ }^{b}$ Expressed in \% of ethanol; ${ }^{\mathrm{c}}$ Expressed in minutes; ${ }^{\mathrm{d}}$ Expressed in mg total polar compounds/g of dry extract; ${ }^{\mathrm{e}}$ Expressed in $\mathrm{mg}$ Gallic acid equivalents/g of dry extract; ${ }^{\mathrm{f}}$ Expressed in $\mathrm{mmol} \mathrm{FeSO}_{4}$ equivalents/g of dry extract; ${ }^{g}$ Expressed in mmol Trolox equivalents/g of dry extract; TPC: Folin-Ciocalteu; FRAP: Ferric Reducing Antioxidant Power; TEAC: Trolox Equivalent Antioxidant Capacity.

\subsection{Multiple Response Optimization Analysis of H. sabdariffa Calyces MAE and L. citriodora Leaves PLE Conditions}

After analyzing the fitting parameters and the effect of each factor in the responses evaluated, the optimization of extraction processes to maximize response variables, phenolic content and bioactivity, was investigated. The antioxidant activity responses revealed different optimal condition depending on the assay to evaluate this bioactivity. For this reason, it was carried out an evaluation of a multiple response analysis with the aim of maximizing both dependent variables, phenolic content and antioxidant capacity. For this analysis, the desirability function was applied. According to Yolmeh and Jafari, the desirability function was based on the idea that the "quality" of a product or process which has multiple quality characteristics; in other words, the desirability function explains the best conditions to achieve the optimum values of evaluated responses [42]. The method finds conditions that provide the "most desirable" response values.

Table 9 shows the Multiple Response Optimization for the extraction of $H$. sabdariffa calyces by MAE and for the extraction of L. citriodora leaves by PLE. In the case of $H$. sabdariffa, the response variable Total Polar Compounds was eliminated and was not considered for this analysis because, as mentioned in previous sections, the adjustment parameters for that response were not adequate. For both matrices, the evaluated model explains the variability of the data for the three response variables evaluated in the case of $H$. sabdariffa, and for the four response variables in L. citriodora, since in both analyses the optimal desirability parameter is about 1.

For H. sabdariffa with the focus on the Multiple Response Optimization to maximize Folin-Ciocalteu, FRAP and TEAC, the proposed optimal conditions were: temperature of $122.25^{\circ} \mathrm{C}$, $\%$ ethanol of $78.58 \%$ and a time of around $3 \mathrm{~min}$. Considering these conditions, the optimal temperature was moderate and the extraction time presented an optimal short time. This can be explained due to the high content of flavonoids present in this matrix, which are thermal sensitive; hence, higher temperatures and extraction times may cause their degradation and, consequently, decrease the antioxidant capacity [17]. The optimal percentage of ethanol proposed by the Multiple Response Optimization was similar to that obtained by Karami et al. for the MAE extraction technique to obtain phenolic compounds from licorice root, which is rich in flavonoids. This study revealed that this percentage of ethanol-water gave an optimal extraction of flavonoids due to the physicochemical characteristics of these solvents [36].

Considering the optimal extraction conditions for Multiple Response Optimization for L. citriodora, the necessary temperature is very low, around $20^{\circ} \mathrm{C}$, extraction time around $7 \mathrm{~min}$ and a very high percentage of ethanol, $95 \%$. This very low temperature may be due to the fact that as the PLE extraction technique uses high pressures, the high pressure enables the solvent penetration in the sample, allowing extraction at lower temperatures and maintaining the stability of the most thermosensitive compounds [43]. Regarding the optimal percentage of ethanol, compounds with high antioxidant capacity, such as verbascoside and its isomers, were extracted with higher ethanol concentrations [44]. 
Table 9. Multiple response optimization analysis in H. sabdariffa and L. citriodora.

H. sabdariffa Multiple Response Optimization with Total Polar Compounds, TPC, FRAP and

TEAC (Optimum Desirability $=1.0$ )

\begin{tabular}{|c|c|c|c|c|c|}
\hline \multicolumn{2}{|c|}{ Independent variables } & \multirow[t]{2}{*}{ TPC } & \multicolumn{2}{|c|}{ FRAP } & \multirow[t]{2}{*}{ TEAC } \\
\hline Temperature $^{a}$ & 122.25 & & \multirow{3}{*}{\multicolumn{2}{|c|}{$0.870^{\mathrm{e}}$}} & \\
\hline Solvent ${ }^{b}$ & 78.58 & \multirow{2}{*}{$78.963^{d}$} & & & $0.249^{f}$ \\
\hline Time $^{\mathrm{c}}$ & 2.98 & & & & \\
\hline \multicolumn{6}{|c|}{$\begin{array}{l}\text { L. citriodora Multiple Response Optimization with TPC, FRAP and TEAC } \\
\text { (Optimum Desirability }=0.99 \text { ) }\end{array}$} \\
\hline \multicolumn{2}{|c|}{ Independent variables } & Total Polar Compounds & TPC & FRAP & TEAC \\
\hline Temperature $^{\mathrm{a}}$ & 19.90 & & & & \\
\hline Solvent ${ }^{\mathrm{b}}$ & 95.05 & $373.449 \mathrm{~g}$ & $252.953^{d}$ & $2.432^{\mathrm{e}}$ & $0.595^{\mathrm{f}}$ \\
\hline Time $^{\mathrm{c}}$ & 6.94 & & & & \\
\hline
\end{tabular}

a Expressed in ${ }^{\circ} \mathrm{C} ;{ }^{b}$ Expressed in \% of ethanol; ${ }^{\mathrm{c}}$ Expressed in minutes; ${ }^{\mathrm{d}}$ Expressed in $\mathrm{mg}$ Gallic acid equivalents/g of dry extract; ${ }^{\mathrm{e}}$ Expressed in mmol $\mathrm{FeSO}_{4}$ equivalents/g of dry extract; ${ }^{\mathrm{f}}$ Expressed in mmol Trolox equivalents/g of dry extract; ${ }^{g}$ Expressed in mg total polar compounds/g of dry extract; TPC: Folin-Ciocalteu; FRAP: Ferric Reducing Antioxidant Power; TEAC: Trolox Equivalent Antioxidant Capacity.

\section{Conclusions}

The extraction conditions using the MAE technique for H. sabdariffa calyces and PLE technique for L. citriodora leaves were optimized to maximize the response variables total phenolic compounds by HPLC-ESI-TOF/MS, TPC by the Folin-Ciocalteu test, and two variables that measure antioxidant capacity, FRAP and TEAC. The quadratic model applied in the H. sabdariffa design represented a very good approximation in the variables of the Folin-Ciocalteu test and in the two antioxidant capacity variables. However, for this matrix, Total Polar Compounds presented a slight deviation to the proposed model. In contrast, the quadratic model proposed in the design for L. citriodora represented a very good approximation for the four response variables evaluated. Through the statistical analysis, it was possible to know the influence of temperature, ethanol percentage and time to allow optimal conditions, maximizing the dependent variables studied. In order to obtain reliable results in the multiple response analysis performed in $H$. sabdariffa, it was deemed appropriate to eliminate the response variable that the model had not adequately adjusted. The results demonstrated that moderate temperature and high ethanol percentage induced the recovery of greater concentration of flavonoids when MAE was applied, achieving better antioxidant results. In the case of L. citriodora, the multiple response analysis included the four study variables. The results revealed that the low temperature and a high percentage of ethanol were needed to obtain high concentration of antioxidant compounds. Considering the evaluated results, it is possible to affirm the importance of multiple response analyses to develop nutraceuticals or functional foods, since it has to be approached from a wide point of view; not only the total content of phenolic compounds or their bioactivity, but also both variables should be evaluated to optimize its production. Furthermore, as confirmed by this study, these analyses can be extrapolated in different techniques and in different matrices with phenolic compounds from different families.

Supplementary Materials: The following are available online at http:/www.mdpi.com/2076-3921/9/12/1175/s1, Table S1: MAE factorial design $2^{3}$ experimental values of tested independent variables, Table S2: PLE factorial design $2^{3}$ experimental values of tested independent variables, Equation (S1): Regression model equations of H. sabdariffa, Equation (S2): Regression model equations of L. citriodora.

Author Contributions: Conceptualization, M.d.1.L.C.-G. and M.d.C.V.-A.; methodology, F.J.L.-J.; software, M.d.C.V.-A. and F.J.L.-J.; validation, M.d.1.L.C.-G.; formal analysis, M.d.1.L.C.-G. and M.d.C.V.-A.; investigation, M.d.C.V.-A. and F.J.L.-J.; data curation, F.J.L.-J. and M.d.l.L.C.-G.; writing-original draft preparation, M.d.C.V.-A.; writing-review and editing, M.d.l.L.C.-G., F.J.L.-J. and D.A.-R. supervision, D.A.-R. and A.S.-C.; project administration, D.A.-R. and A.S.-C.; funding acquisition, D.A.-R. and A.S.-C. All authors have read and agreed to the published version of the manuscript. 
Funding: This research received no external funding.

Acknowledgments: The authors would like to thank the Ministry of Science, Innovation and Universities (RTI2018-096724-B-C22). The author M.d.C.V.-A. gratefully acknowledges the Plan Propio de Investigación of the University of Granada for the fellowship called "Becas de Iniciación a la Investigación" and the Ministry of Science, Innovation and Universities, University of Granada, and AGR274 group for the contract (5450). The author F.J.L.-J. gratefully acknowledges the Spanish Ministry of Economy and Competitiveness (MINECO) for the FPI grant BES-2016-076618.

Conflicts of Interest: The authors declare no conflict of interest.

\section{References}

1. Gul, K.; Singh, A.K.; Jabeen, R. Nutraceuticals and Functional Foods: The Foods for the Future World. Crit. Rev. Food Sci. Nutr. 2016, 56, 2617-2627. [CrossRef]

2. Santos-Lozano, J.M.; Rada, M.; Lapetra, J.; Guinda, Á.; Jiménez-Rodríguez, M.C.; Cayuela, J.A.; Ángel-Lugo, A.; Vilches-Arenas, Á.; Gómez-Martín, A.M.; Ortega-Calvo, M.; et al. Prevention of type 2 diabetes in prediabetic patients by using functional olive oil enriched in oleanolic acid: The PREDIABOLE study, a randomized controlled trial. Diabetes Obes. Metab. 2019, 21, 2526-2534. [CrossRef]

3. Katsube, T.; Yamasaki, M.; Shiwaku, K.; Ishijima, T.; Matsumoto, I.; Abe, K.; Yamasaki, Y. Effect of flavonol glycoside in mulberry (Morus alba L.) leaf on glucose metabolism and oxidative stress in liver in diet-induced obese mice. J. Sci. Food Agric. 2010, 90, 2386-2392. [CrossRef]

4. Sedlak, L.; Wojnar, W.; Zych, M.; Wyględowska-Promieńska, D.; Mrukwa-Kominek, E.; Kaczmarczyk-Sedlak, I. Effect of Resveratrol, a Dietary-Derived Polyphenol, on the Oxidative Stress and Polyol Pathway in the Lens of Rats with Streptozotocin-Induced Diabetes. Nutrients 2018, 10, 1423. [CrossRef]

5. de la Luz Cádiz-Gurrea, M.; Olivares-Vicente, M.; Herranz-López, M.; Román-Arráez, D.; Fernández-Arroyo, S.; Micol, V.; Segura-Carretero, A. Bioassay-guided purification of Lippia citriodora polyphenols with AMPK modulatory activity. J. Funct. Foods 2018, 46, 514-520. [CrossRef]

6. Mezni, A.; Mhadhbi, L.; Khazri, A.; Sellami, B.; Dellali, M.; Mahmoudi, E.; Beyrem, H. The protective effect of Hibiscus sabdariffa calyxes extract against cypermethrin induced oxidative stress in mice. Pestic. Biochem. Physiol. 2020, 165, 104463. [CrossRef] [PubMed]

7. Herranz-López, M.; Olivares-Vicente, M.; Encinar, J.A.; Barrajón-Catalán, E.; Segura-Carretero, A.; Joven, J.; Micol, V. Multi-targeted molecular effects of Hibiscus sabdariffa polyphenols: An opportunity for a global approach to obesity. Nutrients 2017, 9, 907. [CrossRef] [PubMed]

8. Mojiminiyi, F.B.O.; Dikko, M.; Muhammad, B.Y.; Ojobor, P.D.; Ajagbonna, O.P.; Okolo, R.U.; Igbokwe, U.V.; Mojiminiyi, U.E.; Fagbemi, M.A.; Bello, S.O.; et al. Antihypertensive effect of an aqueous extract of the calyx of Hibiscus sabdariffa. Fitoterapia 2007, 78, 292-297. [CrossRef]

9. Ali, R.F.M.; El-Anany, A.M. Hypolipidemic and hypocholesterolemic effect of roselle (Hibiscus sabdariffa L.) seeds oil in experimental male rats. J. Oleo Sci. 2017, 66, 41-49. [CrossRef]

10. Villegas-Aguilar, M.D.C.; Fernández-Ochoa, Á.; Cádiz-Gurrea, M.D.L.L.; Pimentel-Moral, S.; Lozano-Sánchez, J.; Arráez-Román, D.; Segura-Carretero, A. Pleiotropic biological effects of dietary phenolic compounds and their metabolites on energy metabolism, inflammation and aging. Molecules 2020, 25, 596. [CrossRef]

11. Ezzat, S.M.; Salama, M.M.; Seif el-Din, S.H.; Saleh, S.; El-Lakkany, N.M.; Hammam, O.A.; Salem, M.B.; Botros, S.S. Metabolic profile and hepatoprotective activity of the anthocyanin-rich extract of Hibiscus sabdariffa calyces. Pharm. Biol. 2016, 54, 3172-3181. [CrossRef] [PubMed]

12. Leyva-Jiménez, F.J.; Lozano-Sánchez, J.; de la Luz Cádiz-Gurrea, M.; Arráez-Román, D.; Segura-Carretero, A. Functional ingredients based on nutritional phenolics. A case study against inflammation: Lippia genus. Nutrients 2019, 11, 1646. [CrossRef]

13. Rúa, J.; López-Rodríguez, I.; Sanz, J.; del Valle Fernández, P.; Garcia, M.D.C.; Garcia Armesto, M.R. Antimicrobial efficacy of Lippia citriodora natural extract against Escherichia coli and Enterococcus faecalis in "Piel de Sapo" melon juice. Food Sci. Nutr. 2019, 7, 3986-3992. [CrossRef] [PubMed]

14. Leyva-Jiménez, F.J.; Lozano-Sánchez, J.; Borrás-Linares, I.; Arráez-Román, D.; Segura-Carretero, A. Comparative study of conventional and pressurized liquid extraction for recovering bioactive compounds from Lippia citriodora leaves. Food Res. Int. 2018, 109, 213-222. [CrossRef] [PubMed] 
15. Cádiz-Gurrea, M.; Lozano-Sánchez, J.; Fernández-Ochoa, Á.; Segura-Carretero, A. Enhancing the Yield of Bioactive Compounds from Sclerocarya birrea Bark by Green Extraction Approaches. Molecules 2019, 24, 966. [CrossRef] [PubMed]

16. Yu, W.; Chen, H.; Xiang, Z.; He, N. Preparation of Polysaccharides from Ramulus mori, and Their Antioxidant, Anti-Inflammatory and Antibacterial Activities. Molecules 2019, 24, 856. [CrossRef]

17. Pimentel-Moral, S.; Borrás-Linares, I.; Lozano-Sánchez, J.; Arráez-Román, D.; Martínez-Férez, A.; Segura-Carretero, A. Microwave-assisted extraction for Hibiscus sabdariffa bioactive compounds. J. Pharm. Biomed. Anal. 2018, 156, 313-322. [CrossRef]

18. De Biaggi, M.; Donno, D.; Mellano, M.G.; Riondato, I.; Rakotoniaina, E.N.; Beccaro, G.L. Cornus mas (L.) Fruit as a Potential Source of Natural Health-Promoting Compounds: Physico-Chemical Characterisation of Bioactive Components. Plant Foods Hum. Nutr. 2018, 73, 89-94. [CrossRef]

19. Pimentel-Moral, S.; Borrás-Linares, I.; Lozano-Sánchez, J.; Arráez-Román, D.; Martínez-Férez, A.; Segura-Carretero, A. Supercritical $\mathrm{CO}_{2}$ extraction of bioactive compounds from Hibiscus sabdariffa. J. Supercrit. Fluids 2018, 147, 213-221. [CrossRef]

20. Hu, M.; Yan, H.; Fu, Y.; Jiang, Y.; Yao, W.; Yu, S.; Zhang, L.; Wu, Q.; Ding, A.; Shan, M. Optimal extraction study of gastrodin-type components from gastrodia elata tubers by response surface design with integrated phytochemical and bioactivity evaluation. Molecules 2019, 24, 547. [CrossRef]

21. Chen, H.; Xiao, H.; Pang, J. Parameter optimization and potential bioactivity evaluation of a betulin extract from white birch bark. Plants 2020, 9, 392. [CrossRef] [PubMed]

22. Cádiz-Gurrea, M.D.L.L.; Borrás-Linares, I.; Lozano-Sánchez, J.; Joven, J.; Fernández-Arroyo, S.; Segura-Carretero, A. Cocoa and grape seed byproducts as a source of antioxidant and anti-inflammatory proanthocyanidins. Int. J. Mol. Sci. 2017, 18, 376. [CrossRef] [PubMed]

23. Benzie, I.F.F.; Strain, J.J. The Ferric Reducing Ability of Plasma (FRAP) as a Measure of "Antioxidant Power": The FRAP Assay. Anal. Biochem. 1996, 239, 70-76. [CrossRef] [PubMed]

24. Miller, N.J.; Rice-Evans, C.; Davies, M.J.; Gopinathan, V.; Milner, A. A novel method for measuring antioxidant capacity and its application to monitoring the antioxidant status in premature neonates. Clin. Sci. 1993, 84, 407-412. [CrossRef] [PubMed]

25. de la Luz Cádiz-Gurrea, M.; Fernández-Arroyo, S.; Joven, J.; Segura-Carretero, A. Comprehensive characterization by UHPLC-ESI-Q-TOF-MS from an Eryngium bourgatii extract and their antioxidant and anti-inflammatory activities. Food Res. Int. 2013, 50, 197-204. [CrossRef]

26. Skroza, D.; Generalić Mekinić, I.; Svilović, S.; Šimat, V.; Katalinić, V. Investigation of the potential synergistic effect of resveratrol with other phenolic compounds: A case of binary phenolic mixtures. J. Food Compos. Anal. 2015, 38, 13-18. [CrossRef]

27. Sánchez-Marzo, N.; Lozano-Sánchez, J.; Cádiz-Gurrea, M.D.L.L.; Herranz-López, M.; Micol, V.; Segura-Carretero, A. Relationships between chemical structure and antioxidant activity of isolated phytocompounds from lemon verbena. Antioxidants 2019, 8, 324. [CrossRef]

28. Everette, J.D.; Bryant, Q.M.; Green, A.M.; Abbey, Y.A.; Wangila, G.W.; Walker, R.B. Thorough study of reactivity of various compound classes toward the folin-Ciocalteu reagent. J. Agric. Food Chem. 2010, 58, 8139-8144. [CrossRef]

29. Nobossé, P.; Fombang, E.N.; Mbofung, C.M.F. Effects of age and extraction solvent on phytochemical content and antioxidant activity of fresh Moringa oleifera L. leaves. Food Sci. Nutr. 2018, 6, 2188-2198. [CrossRef]

30. Xiao, X.; Song, W.; Wang, J.; Li, G. Microwave-assisted extraction performed in low temperature and in vacuo for the extraction of labile compounds in food samples. Anal. Chim. Acta 2012, 712, 85-93. [CrossRef]

31. Firuzi, O.; Lacanna, A.; Petrucci, R.; Marrosu, G.; Saso, L. Evaluation of the antioxidant activity of flavonoids by "ferric reducing antioxidant power" assay and cyclic voltammetry. Biochim. Biophys. Acta-Gen. Subj. 2005, 1721, 174-184. [CrossRef] [PubMed]

32. Orak, H.H.; Magdalena, K.; Amarowicz, R.; Orak, A.; Penkacik, K. Genotype-Related Differences in the Phenolic Compound Profile and Antioxidant Activity of Extracts from Olive (Olea europaea L.) Leaves. Molecules 2019, 24, 1130. [CrossRef] [PubMed]

33. Sanhueza, L.; Melo, R.; Montero, R.; Maisey, K.; Mendoza, L.; Wilkens, M. Synergistic interactions between phenolic compounds identified in grape pomace extract with antibiotics of different classes against Staphylococcus aureus and Escherichia coli. PLoS ONE 2017, 12, e0172273. [CrossRef] [PubMed] 
34. Gutiérrez Pulido, H.; de la Vara Salazar, R. Análisis y Diseño de Experimentos; Intergovernmental Panel on Climate Change, Ed.; Cambridge University Press: Cambridge, UK, 2004; ISBN 970-10-6526-3.

35. Liyana-Pathirana, C.; Shahidi, F. Optimization of extraction of phenolic compounds from wheat using response surface methodology. Food Chem. 2005, 93, 47-56. [CrossRef]

36. Karami, Z.; Emam-Djomeh, Z.; Mirzaee, H.A.; Khomeiri, M.; Mahoonak, A.S.; Aydani, E. Optimization of microwave assisted extraction (MAE) and soxhlet extraction of phenolic compound from licorice root. J. Food Sci. Technol. 2015, 52, 3242-3253. [CrossRef] [PubMed]

37. Mendes, M.; Carvalho, A.P.; Magalhães, J.M.C.S.; Moreira, M.; Guido, L.; Gomes, A.M.; Delerue-Matos, C. Response surface evaluation of microwave-assisted extraction conditions for Lycium barbarum bioactive compounds. Innov. Food Sci. Emerg. Technol. 2016, 33, 319-326. [CrossRef]

38. Bahorun, T.; Luximon-Ramma, A.; Crozier, A.; Aruoma, O.I. Total phenol, flavonoid, proanthocyanidin and vitamin C levels and antioxidant activities of Mauritian vegetables. J. Sci. Food Agric. 2004, 84, 1553-1561. [CrossRef]

39. Csepregi, K.; Neugart, S.; Schreiner, M.; Hideg, É. Comparative evaluation of total antioxidant capacities of plant polyphenols. Molecules 2016, 21, 208. [CrossRef]

40. DeGraft-Johnson, J.; Kolodziejczyk, K.; Krol, M.; Nowak, P.; Krol, B.; Nowak, D. Ferric-reducing ability power of selected plant polyphenols and their metabolites: Implications for clinical studies on the antioxidant effects of fruits and vegetable consumption. Basic Clin. Pharmacol. Toxicol. 2007, 100, 345-352. [CrossRef]

41. Cesari, L.; Namysl, S.; Canabady-Rochelle, L.; Mutelet, F. Phase equilibria of phenolic compounds in water or ethanol. Fluid Phase Equilib. 2017, 453, 58-66. [CrossRef]

42. Yolmeh, M.; Jafari, S.M. Applications of Response Surface Methodology in the Food Industry Processes. Food Bioprocess Technol. 2017, 10, 413-433. [CrossRef]

43. Tripodo, G.; Ibáñez, E.; Cifuentes, A.; Gilbert-López, B.; Fanali, C. Optimization of pressurized liquid extraction by response surface methodology of Goji berry (Lycium barbarum L.) phenolic bioactive compounds. Electrophoresis 2018, 39, 1673-1682. [CrossRef] [PubMed]

44. Rosa, A.D.; Junges, A.; Fernandes, I.A.; Cansian, R.L.; Corazza, M.L.; Franceschi, E.; Backes, G.T.; Valduga, E. High pressure extraction of olive leaves (Olea europaea): Bioactive compounds, bioactivity and kinetic modelling. J. Food Sci. Technol. 2019, 56, 3864-3876. [CrossRef] [PubMed]

Publisher's Note: MDPI stays neutral with regard to jurisdictional claims in published maps and institutional affiliations.

(C) 2020 by the authors. Licensee MDPI, Basel, Switzerland. This article is an open access article distributed under the terms and conditions of the Creative Commons Attribution (CC BY) license (http://creativecommons.org/licenses/by/4.0/). 